\title{
Assessment of HVDC Grid Segmentation for Reducing the Risk of Cascading Outages and Blackouts
}

\author{
Omid Alizadeh Mousavi, Lazar Bizumic, Rachid Cherkaoui \\ École Polytechnique Fédérale de Lausanne (EPFL)
}

\begin{abstract}
This paper deals with a DC-segmentation of interconnected power systems which represents a relatively new and interesting approach that could be a potential solution for mitigation of the cascading outages effects. The DC-segmentation is defined as the use of DC links to split large $\mathrm{AC}$ grids into sets of smaller asynchronously operated AC segments. By implementing grid segmentation, it should be plausible to improve the controllability of power flows, to limit the propagation of disturbances, to reduce the risk of cascading outages and blackouts and to decrease the complexity of power systems. The statistical analysis of time series of blackouts size measures demonstrates the power law region in distributions of different quantities. This heavy tail behavior implies that the blackouts of different scales may take place and the extreme events cannot be overlooked. In order to take into account the risk of large blackouts, a new risk assessment method is developed and implemented. This method is based on Monte Carlo Simulation and takes into account the cascading outages of transmission system due to the overloading and the hidden failure of protection systems and the response of generators/loads/HVDC controllers to the power imbalance or the frequency deviation. The effectiveness of the DC-segmentation for reducing the risk of large blackouts is assessed using the proposed risk assessment method applied to a simplified model of the potential future intercontinental power grid of Europe and Russia. The distributions and expected values of the blackout size quantities (including Load Not Served (LNS), Number of Transmission component Outage (NTO) and Energy Not Served (ENS)) are evaluated before and after the DCsegmentation.
\end{abstract}

\section{1- Introduction}

The modern power transmission networks are large and complex systems that have experienced wide blackouts in the recent two decades (i.e. the Northeast and Italy blackouts in 2003 and India 2012). These rare but significant events increase concerns for the stable and secure operation of power systems. The main challenges for secure operation of power systems are: complex modes of instability (angle, voltage and frequency), propagation of disturbances across $\mathrm{AC}$ grids and vulnerability to sabotage strategies. In order to keep a complex power system stable and secure, operators need to closely coordinate many processes. However, the electricity market and its deregulation are pushing the electric power industry from cooperation and coordination to competition and confidentiality [1]. In this respect, system operators confront with a challenging problem for appropriate and coordinated provision of security control actions regarding the balance between the economy and security. The new rules and procedures have led to increased complications in power systems operation and control [2].

An additional aspect that increases the complexity of the problem is the operation of single power systems within an interconnected continental-scale grid (e.g. interconnected networks in continental Europe ENTSOE and North America - NERC). The areas of an interconnected power system generally profit from (i) increased security and (ii) mutual economically efficient generation. However, it is shown in [3] that the security of the resulting interconnected power system could decrease with the increase of the interconnection's size and consequently the complexity of the system.

In this situation, the traditional approaches for increasing the transmission network capacity do not inherently enhance the security of the power system because security of a system is also determined by the rules that govern its operation [4]. An effective approach should develop a defense plan against extreme contingencies and must achieve immunity against the large scale blackouts. Also, it should act in complex situations and improve the controllability to survive inevitable disturbances with minimum interruption of customer service.

In order to achieve those goals, increasingly popular and capable power electronic devices could be used. In this respect, HVDC and FACTS technologies can deal with growing uncertainties and increasing complexities of 
power systems. From the reliability and economic point of view, these components can play a significant role in the power systems [5].

One of the most important characteristics of HVDC is that DC links can dynamically insulate a part of the grid from the rest of the system resulting in improved grid performance. In contingency conditions, unlike AC lines, DC links would not be overloaded due to the controllability of power flows which significantly reduces the risk of cascading outages [6]. Tanks to this, they can separate parts of interconnected systems to avoid the widening of large disturbances through the system [5]. In general, the DC links provide reliable support and performance enhancement for the surrounding AC grid [7]. Based on the current operational experience, the effective performance of the existing DC links as a firewall against the cascading outages during major disturbances is reported. For instances, the DC links between Quebec and the disturbed area in the Northeast 2003 blackout [8] and also between the southern part and the rest of grid of India in 2012 [9], stopped the propagation of the cascading outages at the asynchronous interfaces. According to the experiences obtained from the large blackouts, DC links could play an important role in the future system development [10].

In order to effectively exploit and maximize all the benefits of the HVDC technology, an interesting idea of power system DC-segmentation has been proposed in literatures [2] and [11]-[13]. The DC-segmentation is defined as the use of DC links to segment large AC grids into sets of asynchronously operated AC segments [11]. Figure 1, shows the concept of the DC-segmentation of the large AC system into several smaller AC segments interconnected solely by means of DC links.

As shown in Fig. 1, the DC links can be bi/tri-polar conventional and/or VSC-based HVDC (in blue) or Backto-Back HVDC (in red) or Multi-Terminal HVDC (in yellow). Usually, the DC-segmentation can be accomplished by conversion of existing AC lines to DC links. Additionally, the converted AC lines would also have higher transfer capabilities than before conversion [10]. Therefore, there would not be significant additional costs for the transmission lines, except the ones associated with $\mathrm{AC} / \mathrm{DC}$ converter stations. Additionally, in order to reduce segmentation costs and construction of new convertor stations, the existing DC links can be considered as a part of a DC-segmentation scheme. An approach presented in [14] can be used in order to determine the costs of DC-segmentation. Moreover, this reference proposes a method for optimal operation of a segmented grid through inter-market optimization and scheduling process between the segments.

One of the crucial questions regarding the grid segmentation is a determination of the optimal cluster sizes and borders. These boundaries of DC-segmentation could be formed according to several criteria such as: location of existing DC links, stability limited paths, congested paths that need more transfer capability, the system security and reliability indices, and political boundaries where it is applicable. The appropriate allocation of DC links requires for a multi-aspect investigation which takes into account these criteria. In practice, system planers and operators have good ideas about potential separation boundaries and number of islands under both, technical and nontechnical concerns [15].

It is worth to mention that the FACTS devices cannot be proposed as a substitute to interconnect the segments because in this case the two side segments still remains synchronous. Moreover, the control of the added FACTS devices to the system may increase the complexity of the system. However, the FACTS technology could play its role inside the new, smaller synchronous interconnections of large power system segments [7]. The feasibility of the segmentation with energy storage technology, instead of DC links, is studied in [16]. Although the segmentation using energy storage devices, allocated at the tie line


Fig. 1. DC-Segmentation of power system contains; a) division of the AC system into segments and b) reconnection of the segments through DC links. 
terminal buses, improves dynamic stability performance of the system, the interconnected system still remains synchronous and sensitive to the large vulnerabilities.

The main advantages of the DC-segmentation for the power grid are the increase of controllability and the decrease of complexity. This finally results in increase of transfer capacity and market efficiency and in decrease of stability constraints and risk of cascading outages. The alignment of the contract paths and the physical (actual) paths of power flow becomes easier since several technical limitations (e.g. stability limitations and transfer capability) are less constraining and the quantity of controllable power is increased. Therefore, the difficulties for efficient electricity market management and development decrease.

In a segmented system, the conventional benefits of the interconnected system such as the generation and reserve sharing and emergency responses are improved or they can be more precisely scheduled. Using the proper HVDC control strategies, the segments can provide as much assistance as plausible for each other during disturbances. However, the DC link controllers can limit the flow of power to the disturbed segment whenever it jeopardizes the supporting segments. As a result, cascading outages and collapse will be limited to only one segment which reduces the risk of widespread blackouts [12]. Owing to this peculiarity, literatures introduce the DC-segmentation as a solution for softening the blow of disturbances [13]. It is also entitled as the grid shock absorber [13] and firewall against disturbances [17]. Therefore, the DCsegmentation provides major improvements in bulk grid security and reliability through the elimination of widespread cascading blackouts [12]. However, it does not solve all of the security concerns and reliability problems. Nevertheless, these issues would be more localized, less complex and hence easier and more effective to be addressed [7].

Literatures investigate the advantages of DCsegmentation for enhancement of the voltage controllability specifically in case of VSC-HVDC [11] and for improvement of the angular stability as in [18] and [19]. Reference [19] demonstrates that the angular stability is improved more in the segmented system than in the system with multi-infeed HVDC without segmentation. Additionally, the application of DC links in the segmentation inherently reduces the fault current level and its negative consequences will be decreased. Regarding the frequency control, two control modes are proposed in [20] for the participation of DC links which are a) fixed active power, and b) active power control using the frequency difference. The proposed control in [18] adjusts the active power of DC links proportional to the frequency deviation of the post-contingency state with respect to two lower/upper limits for frequency deviation. Furthermore, [21] studies the coordination of the protection and VSC-HVDC systems to improve the system security in the post-contingency state and [22] proposes the coordination of the DC link frequency control with existing underfrequency load shedding schemes to limit frequency decay during a major disturbance. As a result, it is suggested that the DCsegmentation can limit the cascading failures and collapse to only one segment which reduce the risk of large blackouts [12].

The literature survey demonstrates the lack of a quantitative study of the DC-segmentation advantages for reducing the risk of cascading outages and large blackouts. This paper focuses on this important aspect using a statistical approach to investigate the risk of cascading outages and large blackouts. The structure of the paper is as follows. In the next section a detailed statistical analysis is performed on the time series of the blackouts data of ENTSO-E to investigate the peculiarities of their distributions. Then, the risk assessment method that captures the cascading outages, the response of frequency control reserves and the model of DC links is presented. In section IV, the proposed risk assessment approach is applied to the potential future intercontinental network of Europe/Russia before and after the DC-segmentation. Finally, Section V discusses the main outcomes of this study.

\section{2- Analysis of Blackouts Data}

The study of the time series of blackouts size measures like Energy Not Served (ENS), Load Not Served (LNS) and number of unserved customers has shown a power law region in their distributions [23]. In this paper, a detailed statistical analysis is performed on the blackouts data of the European transmission network for three reliability indicators considered by ENTSO-E, which are ENS, LNS and Restoration Time (RT). The data are available for major fault events between January 2002 and June 2012 [24].

Several studies worldwide have shown a power law region in the distribution of the blackout size larger than a given size. Here, the maximum likelihood approach is used to estimate the power law function and the Kolmogorov-Smirnov (KS) statistic is used to test the goodness-of-fit [25]. The probability distributions of three measures and their maximum likelihood power law fit are shown in Fig. 2. The generic statistical measures including the number of events $(n)$, mean $(\bar{x})$, standard deviation $(\sigma)$ and maximum observed occurrences $\left(x_{\max }\right)$ are given in the left side of Table 1. The right side of Table 1 shows the power law fits including the lower 

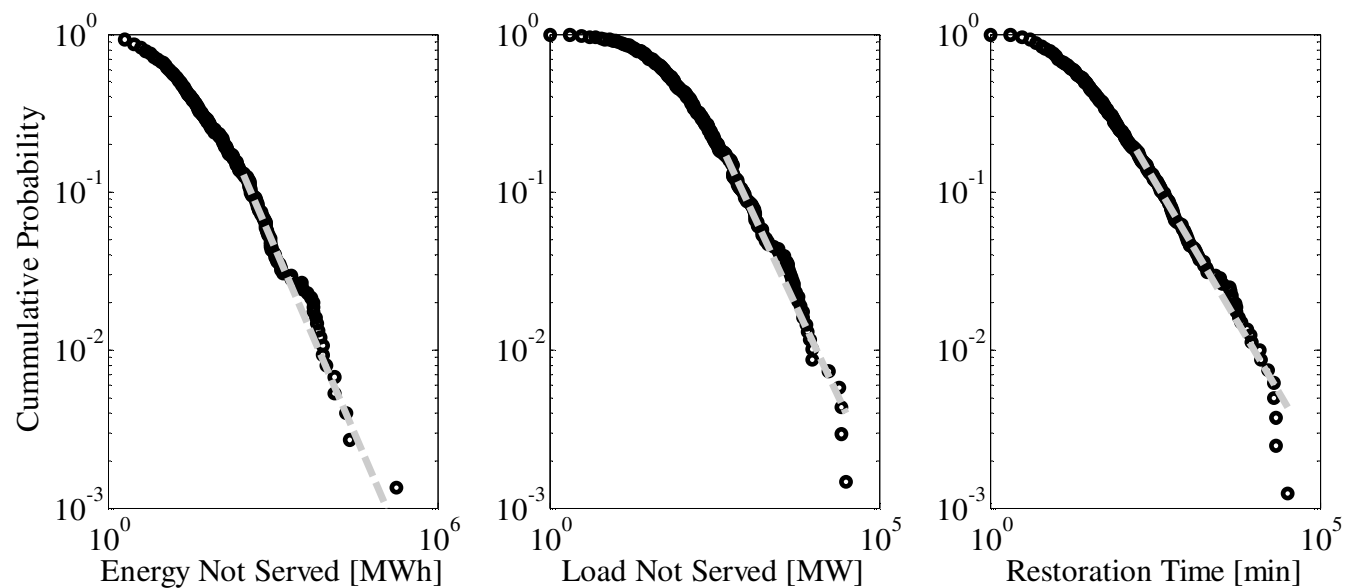

Fig. 2. Cumulative distribution functions (black circles) and their maximum likelihood power law fit (gray dashed line) for ENTSOE reliability quantities from January 2002 to June 2012.

Table 1. Statistics of major events in ENTSOE from January 2002 to June 2012.

\begin{tabular}{|c|cccc|cccc|}
\hline Data Set & $n$ & $\bar{x}$ & $\sigma$ & $x_{\max }$ & $x_{\min }$ & $\alpha$ & $n_{\text {tail }}$ & $p$ \\
\hline ENS & 747 & 559.71 & 6773.6 & 180000 & $300 \pm 154$ & $1.81 \pm 0.11$ & $94 \pm 116$ & 0.495 \\
LNS & 686 & 584.84 & 2438.4 & 31990 & $478 \pm 141$ & $1.89 \pm 0.10$ & $115 \pm 80$ & 0.372 \\
RT & 807 & 407.99 & 2178.2 & 32126 & $163 \pm 52$ & $1.71 \pm 0.06$ & $148 \pm 92$ & 0.935 \\
\hline
\end{tabular}

band to the power law behavior $\left(x_{\text {min }}\right)$, scaling parameter $(\alpha)$, the number of occurrences in the power law region $\left(n_{\text {tail }}\right)$ and $\mathrm{p}$ value $(p)$.

A similar approach is applied in [26] to the ENTSO-E major event data from 2002 to 2008. The obtained statistical results in Fig. 2 and Table 1 are consistent with the presented results in [26] whereas our results profit the longer time series of blackouts data until July 2012. It is worth to mention that these statistical properties are obtained for a power grid whose topology continuously evolves over the time. However, a power system with a fixed topology (that is sufficiently large) is also expected to have an approximate power law over some range of values.

The power law region demonstrates that the dynamics of blackouts can be associated to complex systems with SelfOrganized Criticality (SOC) feature [23]. In the system with the SOC characteristic, there are different types of variables with opposing driving forces that, in certain conditions, could drive the system into a critical operation state. Furthermore, the power law region in the distribution implies that the blackouts of different scales may take place and the extreme events cannot be overlooked. The system may experience large blackouts with certain probabilities and the occurrences of small/large blackouts are not independent but correlated each other (e.g. [27], [28]). Therefore, a proposed blackout risk assessment method should quantify the power law region with reference to different quantities of blackout size [27].

\section{3- Risk Assessment Approach}

For the risk assessment purpose, this section describes a statistical method aimed at numerically evaluating the risk of cascading blackouts. In this respect, the Monte Carlo Simulation (MCS) is applied to provide contingency scenarios including both generation and transmission outages (AC lines and DC links). A random variation in the loading pattern is considered while the total load is kept constant. The reason for this is that the distribution of loads changes during the day and between days and cascading blackouts could start at different random loading patterns. The random loading pattern is modeled with a normal distribution whereas the mean is equal to the amounts of the loads and $5 \%$ of the variance is also considered.

For a given scenario, the method considers the following elements:

a) the effects of cascading outages due to overloads and hidden failure of protection systems,

b) the response of the automatic Frequency Control Reserve (FCR) of the generating units and the selfregulation of loads to power imbalance (i.e. frequency deviation) in each step of cascading outages,

c) the response of DC links inverter and rectifier to the power imbalance,

d) the operator intervention as the corrective actions after several steps of cascading outages using manual and automatic FCRs and load shedding. 
The contingency scenarios construct the system states and select the initial events. In the simulation procedure, after initial events or after each step of cascading outages, there may be a power imbalance and, consequently, a frequency deviation in the system. A distributed slack bus model is employed in such a way that all of the remaining dispatched generating units share the power imbalance according to their droop frequency-control characteristics $\left(R_{g}\right)$ and their residual capacity where $\bar{P}_{g}\left(P_{g_{0}}\right)$ is the maximum (initial) power output of generators and $P_{g_{A}}$ is the automatic FCR of generators. Also the effect of selfregulation of loads $\left(D_{d}\right)$ is taken into account [29]. The response of DC links inverter and rectifier to the frequency deviation are modeled with the generators droop $\left(R_{I n v}\right)$ and the load frequency regulation $\left(D_{R e c}\right)$, respectively. It should be noted that the responses of DC links are limited to their upper/lower transfer capability limits. Therefore, the frequency deviation $(\Delta f)$ of the system in each step of cascading outages is calculated according to the following equation:

$$
\Delta f=\frac{-\Delta P}{\sum_{\Omega_{D}} D_{e q}^{i}+\sum_{\Omega_{G}} \frac{1}{R_{e q}^{i}}}
$$

where $\Delta P$ is the power imbalance in $M W . D_{e q}^{i}$ and $R_{e q}^{i}$ are the equivalent frequency characteristics of $i$-th bus obtained by (2) and (5) in $\mathrm{MW} / \mathrm{Hz}$ and $\mathrm{Hz} / \mathrm{MW}$, respectively. $\Omega_{D}$ (resp. $\Omega_{G}$ ) indicates the set of demands and rectifiers (resp. generators and inverters). For the set of generators and inverters connected to $i$-th bus $\left(\Omega_{G, i}\right)$ the equivalent droop $\left(R_{e q}^{i}\right)$, the equivalent initial active power generation $\left(P_{g_{0}, e q}^{i}\right)$ and the equivalent maximum active power generation $\left(\bar{P}_{g, e q}^{i}\right)$ are calculated by (2), (3) and (4), respectively.

$$
\begin{gathered}
\frac{1}{\mathrm{R}_{e q}^{i}}=\sum_{\Omega_{G, i}}\left(\frac{1}{\mathrm{R}_{g}^{i}}+\frac{1}{\mathrm{R}_{I n v}^{i}}\right) \\
P_{g_{0}, e q}^{i}=\sum_{\Omega_{G, i}}\left(P_{g_{0}}^{i}+P_{I n v_{0}}^{i}\right) \\
\bar{P}_{g, e q}^{i}=\sum_{\Omega_{G, i}}\left(\bar{P}_{g}^{i}+\bar{P}_{I n v}^{i}\right)
\end{gathered}
$$

where $\bar{P}_{I n v}^{i}$ (resp. $P_{I n v_{0}}^{i}$ ) is the maximum (resp. initial) power of the DC links. Similarly, for the set of loads and rectifiers connected to $i$-th bus $\left(\Omega_{D, i}\right)$ the equivalent droop $\left(D_{e q}^{i}\right)$ and the equivalent demand $\left(P_{d, e q}^{i}\right)$ are calculated by (5) and (6), respectively.

$$
\begin{gathered}
D_{e q}^{i}=\sum_{\Omega_{G, i}}\left(\frac{P_{d_{0}}^{i} \cdot D_{d}^{i}+P_{\operatorname{Re} c_{0}}^{i} \cdot D_{\operatorname{Re} c}^{i}}{P_{d_{0}}^{i}+P_{\operatorname{Re} c_{0}}^{i}}\right) \\
P_{d_{0}, e q}^{i}=\sum_{\Omega_{D, i}}\left(P_{d_{0}}^{i}+P_{R e c_{0}}^{i}\right)
\end{gathered}
$$

where $P_{R e c_{0}}^{i}$ is the initial power of DC links. It is worth mentioning that for different loading patterns, a linear load flow (DCLF) is used to initially calculate $P_{I n v_{0}}^{i}$ and $P_{\text {Rec }}^{i}$ in pre-contingency state.

After the generation and load balance is restored, a DCLF is applied to calculate the power flow in each step of the cascading outages. For AC lines, the effect of transmission system overloads and hidden failure of protection systems in cascading outages are considered with a flow-dependent probability as given in [3].

In reality, during the emergency state the system operator has an opportunity to implement some corrective actions and minimize the amount of lost load, after several steps of cascading outages. In this simulation, a model of operator's response to contingencies is considered as a linearized Optimal Power Flow (DCOPF). The aim of the DCOPF is the minimization of the lost load through redispatching the generating units and shedding some loads. It is assumed that in this step the operator can utilize both automatic and manual FCRs $\left(\bar{P}_{g}=P_{g_{0}}+P_{g_{A}}+P_{g_{M}}\right)$ where $P_{g_{M}}$ is the manual FCR of generators. In [29] investigation on the cascading dynamics of the power system blackouts shows that this model is appropriate to demonstrate the power law region in the blackouts size distribution. Note that the presented simulation approach studies a power system with a fixed topology. Therefore, the continuous evolution of the power system state and configuration under complex dynamics are, in general, neglected [30].

After simulating each scenario, the obtained amount of lost loads (LNS) and the Number of Transmission component Outages (NTO) are used to evaluate the risk of blackout. For this purpose, the Complementary Cumulative Distribution Function (CCDF) of the continuous random variables (e.g. the lost load) is calculated by simply ranking the data and then scaling the ranked data. Also, the Probability Distribution Function (PDF) of discrete random variables (e.g. the NTO) is calculated simply by assigning a probability to each possible value such that the total probability for all random variables is equal to 1 . The sufficient number of the MCS samples is calculated to ensure a specific level of accuracy associated with a confidence level [3]. The accuracy level is used as the stopping criteria for the MCS. It is selected by qualitatively analyzing smoothness of distributions particularly in their tail behaviors. At the end of the MCS trials, expected values (e.g. Expected LNS (ELNS) and Expected ENS (EENS)) are calculated using mean operator of the random variable.

In order to quantify the value of risk in terms of cost, the ENS should be assessed. For each scenario, the ENS can be obtained by multiplying the amount of the lost load 
and the restoration time. The amount of lost load is obtained directly from the simulation results. The number of transmission outages has a direct relation to the restoration time. So it is assumed as an indicator for the restoration time. Interrupted Energy Assessment Rate (IEAR) in $\$ / k W h$ is introduced in [27], that is a factor multiplying the ENS to estimate the overall blackout cost. This cost is assumed to be fixed in [31] (IEAR $=5.27$ $\$ / \mathrm{kWh}$ ) while indeed there is a nonlinear relationship. Since this reference has been published in 1987 the IEAR value should be calculated in present value. By taking into account the inflation over a period of 26 years, adjusted present value of IEAR is around $7.5 € / \mathrm{kWh}$ at present time.

It should be noted that this risk assessment method is based on DC model of the transmission system (DCLF and DCOPF) which can cover only some of the most important phenomena associated to blackout dynamics. Therefore, the risk of cascading failures leading to voltage excursions and collapse and transient instability are not taken into consideration. As mentioned earlier in section 2, the DC-segmentation generally improves the voltage and angular stability. Besides, this paper only studies the risk of cascading outages and system frequency deviation where these two stability mechanisms do not impose high impact. From now whenever the term segmentation is used, it refers to the DC-segmentation.

\section{4- Simulation and Discussion}

The effect of the segmentation on the risk of cascading outages and blackouts are investigated on a simplified model of the potential future intercontinental EuropeRussian network. Initially, it is assumed that the network of Europe and Russia are interconnected with AC lines. The schematic of the studied grid is shown in Fig. 3. The $\mathrm{AC}$ lines and the existing DC links are displayed in black and red, respectively. Note that each AC line (resp. node with generator) in Fig. 3 may represent several parallel lines (resp. generating units connected to a node). The total load of the system is 9324.75 p.u. and the total installed capacity of generating units is equal to 16419.30 p.u., where per unit parameters are on a 100 MVA base. Furthermore, the amounts of automatic and manual FCRs are assumed equal to $7 \%$ and $10 \%$ of the total capacity of generators, respectively. It is assumed that they are evenly distributed based on the net generation and consumption and the remained capacity of each generator. Note that according to the hierarchical FCR (with primary, secondary and tertiary), the automatic FCR can be considered as the primary and part of the secondary whereas the manual FCR is composed of the remained part of the secondary and the tertiary.

The unavailability of transmission lines and generation units are assumed equal to 0.01. The response of generator $\left(r_{g}\right)$ and loads $\left(d_{n}\right)$ to the frequency deviation are considered equal to 0.05 and 1.00 , respectively. Note that $r_{g}$ and $d_{n}$ are in per-unit and they are converted to $R_{g}$ and $D_{n}$ for each generator and load by using equation (1). It is worth to mention that the studied grid is assumed $N-1$ secure (resilient to single outage of generating units and transmission lines) that is commonly kept by the system operators in the operation of power systems.

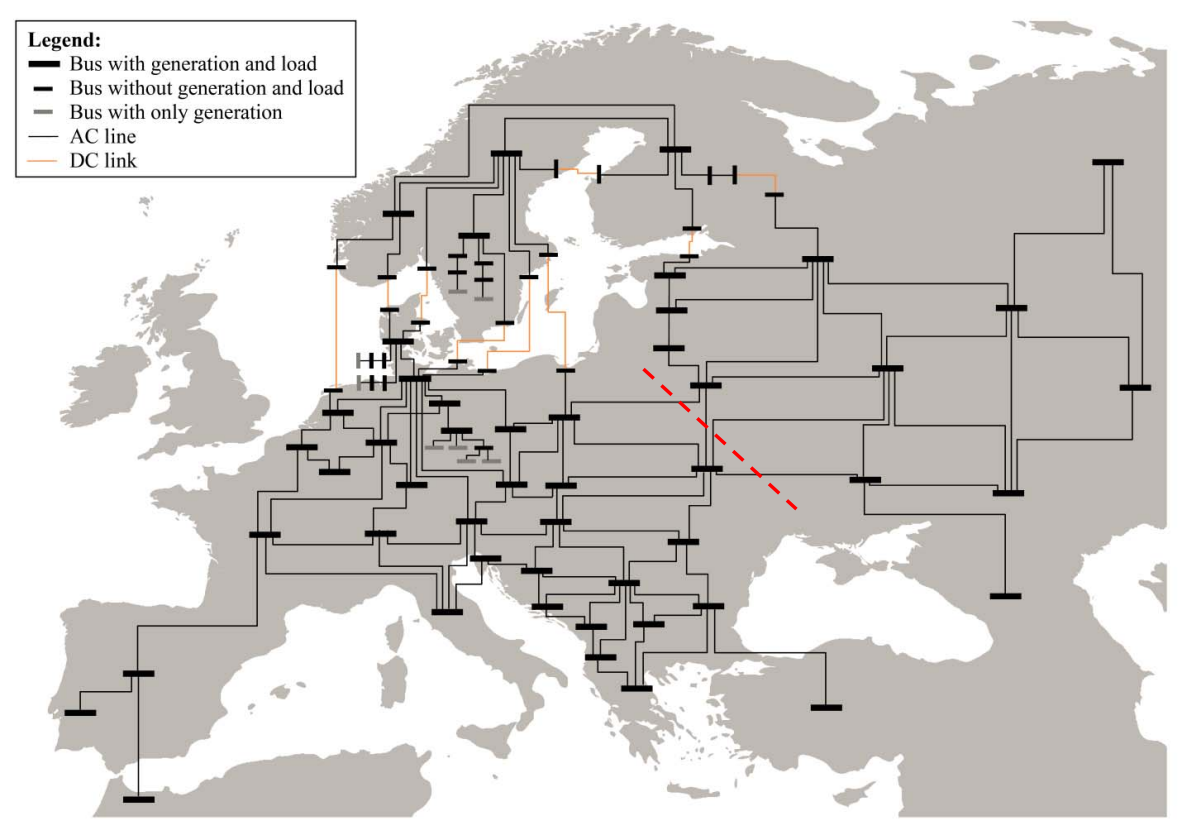

Fig. 3. The schematic of the simplified model of the future Europe-Russian network. 
The proposed segmentation scheme divides the system into three segments. This scheme profits the existing Nordel segment (network of northern Europe) that is connected to the rest of the grid only with DC links. These DC links are shown with red solid lines. The location of newly added DC links is shown with a red dashed line in Fig. 3. These DC links are located at the interconnection between the network of Europe and Russia which represent a possibility for interconnecting the networks only with DC links.

The proposed blackout risk analysis method is applied for two cases, a) the system with AC lines as interconnections and b) the system with DC links in place (segmented system). Then, the risks of blackout before and after segmentation are compared using the distribution of the LNS, the ENS and the NTOs as the measures of the blackout size. For this purpose Fig. 4, 5 and 6 show the CCDF of the LNS data, the CCDF of the ENS data and the PDF of the NTOs, respectively. The CCDFs and the PDF are plotted in the log-log and log-linear axes, respectively, to illustrate the power law regions and behaviors in the distributions.

As Fig. 4 shows, the PDF of the NTO before segmentation has a heavy tail for the large number of outages. However, after segmentation not only the probability of the large number of outages (larger than 7 outages) decrease but also the maximum number of outages is limited to 22 that is quite lower than the case before segmentation (with 32 outages). As a result, the segmentation effectively decreases the probability of the heavy tail region in the PDF of NTO.

Fig. 5 and Fig. 6 show the CCDF of LNS and ENS, respectively. The CCDFs of the LNS have similar behavior before and after segmentation. However, for LNS larger than 100 p.u. the case without segmentation has events with larger amounts of LNS. The ELNS is decreased from 71.42 MW (before segmentation) to 69.64 MW (after segmentation).

The CCDFs of the ENS in Fig. 6 show that the segmentation effectively decreases the size of the largest blackouts. The largest blackout size before the segmentation is 7075 p.u. ( $707500 \mathrm{MWh})$ while it is reduced to 920 p.u. $(92000 \mathrm{MWh})$ after the segmentation. Moreover, the segmentation has decreased the EENS from 90.03 $\mathrm{MWh}$ (before segmentation) to $73.90 \mathrm{MWh}$ (after segmentation).

Interestingly, the major change in the distribution of blackout size occurs in the heavy tail region corresponding to the large blackouts. The simulation results effectively show a shift in the distribution of this region toward lower blackout sizes. As a result, the

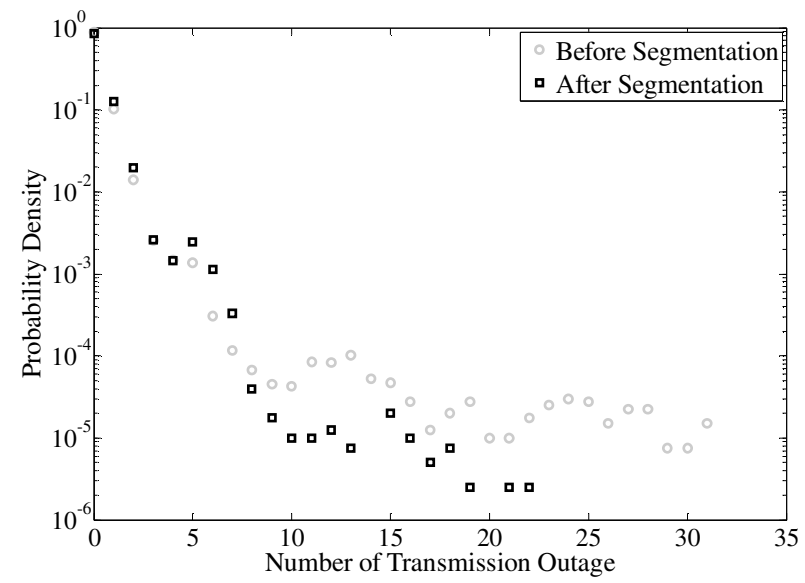

Fig. 4. PDF of number of transmission component outage (NTO) before and after segmentation.

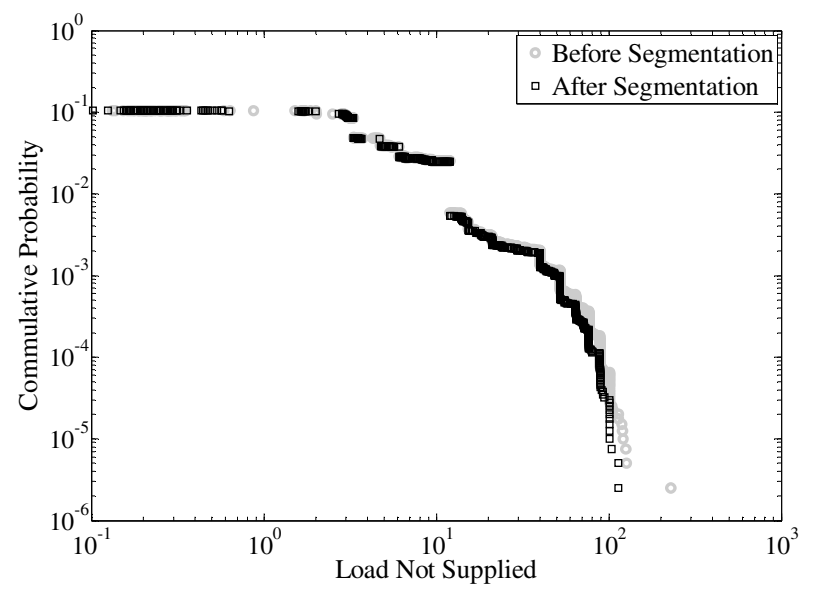

Fig. 5. CCDF of load not supplied (LNS) before and after segmentation.

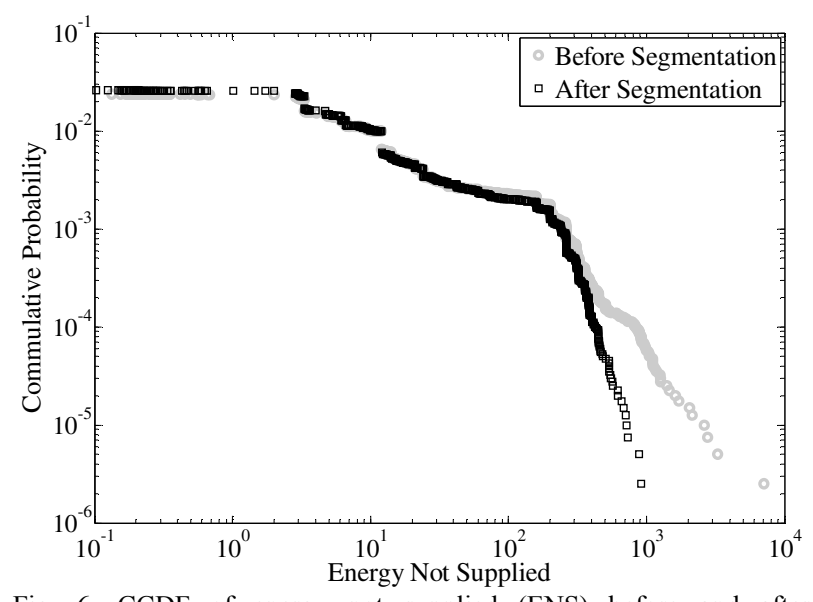

Fig. 6. CCDF of energy not supplied (ENS) before and after segmentation.

segmentation can be considered as a technically appropriate approach to restrain the propagation of the cascading outages and consequently the widespread large blackouts. 
The presented results so far consider that the DC link controllers are in the constant power output control mode. The effect of DC links responses to the power imbalance is investigated through specifying different droop values to the DC link controllers in response to the frequency deviations, as specified in equations (2)-(6). For this purpose three cases are studied, namely (i) without droop response, (ii) low droop response and (iii) high droop response. The first case considers constant power output for DC links as it is mentioned as "after segmentation" in the already presented results. In the second case the response of the DC links frequency control is selected similar with the typical response of generators/loads. The third case assumes ten times higher response than the second case. Using this control, if frequency in one cluster starts to decline (e.g. due to generation loss), bordering DC links will adjust their power transfers according to their controllers characteristics and the frequency difference between the clusters. In this way, it is plausible to benefit from the help of neighboring clusters in emergency states.

The distribution of NTO, LNS and ENS are depicted for three cases in Fig. 7, 8 and 9, respectively. As shown in Fig. 7, the probability of large NTO as well as the maximum NTO increases with the increase of DC link response. Figure 8 does not show a distinct difference between CCDF of LNS for different cases. The CCDF of ENS in Fig. 9 shows again that the region related to large blackouts shifts toward larger blackout sizes with the increase of DC link response.

Table 2 summarizes the ELNS and EENS before and after segmentation for different cases. The expected values increase with the increase of the DC link responses which is consistent with the obtained results from the distributions. Therefore, in the segmented power system the increase of DC links responses to the frequency deviation, as it is modeled here, increase probability of large blackouts. Moreover, the expected values of the case before segmentation effectively decrease after segmentation in the cases without response and with low response of DC links. However, in the case of after segmentation with high response of DC links, the ELNS and EENS become higher than in the case before segmentation. This can be explained by the fact that the local response of DC link controllers to the frequency deviation can cause some of the clusters internal AC lines to overload and trip. This way, the contingencies can still pass from one cluster to the neighboring ones, which is not acceptable.

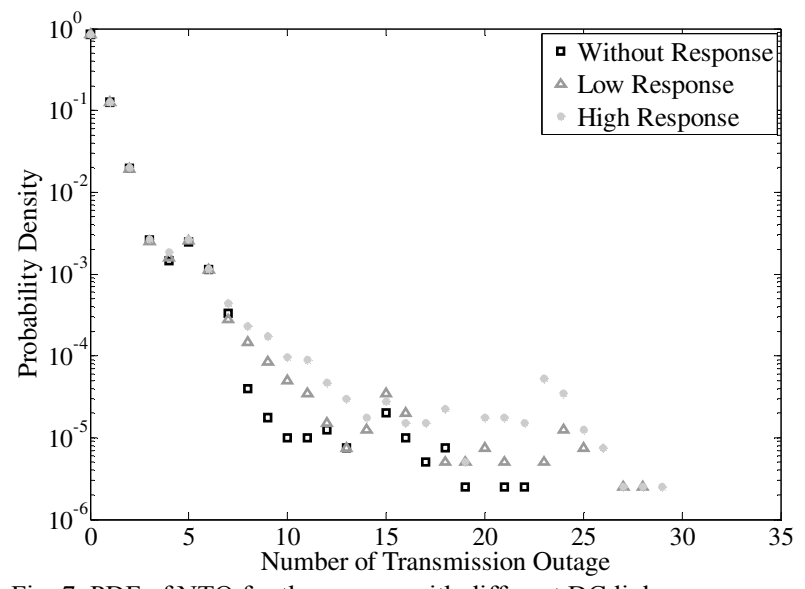

Fig. 7. PDF of NTO for three cases with different DC link responses.



Fig. 8. CCDF of LNS for three cases with different DC link responses.

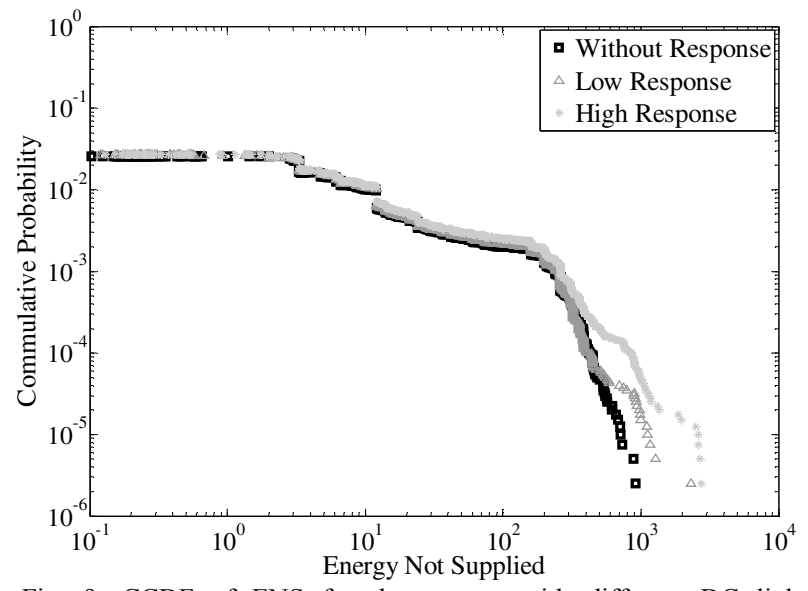

Fig. 9. CCDF of ENS for three cases with different DC link responses.

Table 2. The ELNS and EENS for different cases.

\begin{tabular}{|c|cccc|}
\hline & before segmentation & \multicolumn{3}{|c|}{ after segmentation } \\
\cline { 2 - 5 } & & without response & low response & high response \\
\hline ELNS (MW) & 71.42 & 69.64 & 70.39 & 72.57 \\
\hline EENS (MWh) & 90.03 & 73.90 & 79.30 & 100.18 \\
\hline
\end{tabular}


It should be noted that this model assumes that the DC link controllers respond based only on their observed frequency deviation in a decentralized and noncoordinated manner. In order to significantly improve effects of the local HVDC control and avoid abovementioned problems, one may propose a coordinated and centralized approach for obtaining the optimal response of DC links in response to severe contingencies. It can be expected that in this case, the achieved results will be even better than the case without the response of DC controllers.

\section{5- Conclusion and Future Investigations}

The statistical analysis of the blackouts indicators in the network of Europe demonstrates that the probability of large blackouts cannot be overlooked. In response to these large blackouts, the DC-segmentation has been proposed in the literatures to confine the propagation of cascading outages to the originating subsystem. A risk assessment method is proposed in order to quantitatively study the effectiveness of DC-segmentation for reducing the risk of large blackouts. The proposed risk assessment approach is able to capture the risk of large blackouts. It takes in to account the cascading outages of transmission system due to the overloading and the hidden failure of protection systems and the response of generators/loads/HVDC controllers to the frequency deviation. The simulation results for the possible future inter-continental grid of Europe and Russia, before and after DC-segmentation, demonstrate that the risk of cascading outages and large blackouts can be decreased effectively. Moreover, the consequence of high response of DC links to the power imbalance on the increase of the probability of large blackouts is demonstrated. A more detailed model of the DC link controllers with additional control responsibilities (e.g. minimization of the transmission system overloading after occurrence of a contingency) can be proposed to exploit more advantages from the DC-segmentation.

The possibility for further segmenting the existing continental grid of Europe/Russia into smaller segments can also be investigated. In this respect, several DCsegmentation configurations can be proposed for replacing a set of $\mathrm{AC}$ lines with $\mathrm{DC}$ links, and then the presented risk assessment approach can be applied to evaluate the risk of each configuration. This study requires a more detailed model of the network. Besides, in the context of evolving power systems under complex dynamics, a Highly Optimized Tolerance (HOT) model (presented in [32]) can be proposed to design the system with the complexity and power laws in uncertain environments. It a-priori assumes that there is a relationship governing how the resources in the system act to limit the event size. In this optimization model the
DC links can be treated as the resources. The HOT state is obtained by minimizing the expected cost of the events subject to the constraints of the resources. In this way, greater resources are devoted to high probability regions of distribution.

\section{References}

[1] G. C. Loehr, "And There Will Be Blackouts", American Education Institute, 2000

[2] G. C. Loehr, "Enhancing the Grid, Smaller Can Be Better," Energybiz magazine, pp. 35-36, Jan/Feb 2007.

[3] O. Alizadeh Mousavi and et. all, "Investigation of the Blackouts Complexity Regarding Spinning Reserve and Frequency Control in Interconnected Power Systems," in PMAPS, Istanbul, 2012.

[4] D. S. Kirschen, "Do Investments Prevent Blackouts?," in IEEE Power Engineering Society General Meeting, 2007.

[5] D. Povh, I. Pyc, D. Retzmann and M. G. Weinhold, "Future Developments in Power Industry", Krakow: $4^{\text {th }}$ IERE General Meeting, 2004

[6] G. Andersson and et. all, "Causes of the 2003 major grid blackouts in North America and Europe, and recommended means to improve system dynamic performance," IEEE Transactions on Power Systems, vol. 20, no. 4, pp. 1922-1928, 2005.

[7] "A layman's guide to power networks - Reliable electricity networks, from power generation to the plug," 4 June 2012. [Online]. Available: http://www.abb.com/cawp/.

[8] P. Bahrman and B. K. Johnson, "The ABCs of HVDC Transmission Technologies," IEEE power \& energy magazine, pp. 32-44, march/april 2007.

[9] G. Runte, "India's Outages: What Can We Learn?," The energy collective, 10 August 2012. [Online]. Available: $\mathrm{http}: / /$ theenergycollective.com/gerry-runte/102106/india-s-outageswhat-can-we-learn.

[10] W. Breuer, D. Povh, D. Retzmann and E. Teltsch, "Trends for future HVDC applications," in $16^{\text {th }}$ Conference of the Electric Power Supply Industry, Mumbai, 2006.

[11] H. Clark and D. Woodford, "Segmentation of the Power System with DC Links", IEEE HVDC-FACTS Subcommittee Meeting, 2006.

[12] H. K. Clark, M. M. El-Gasseir and H. D. Kenneth Epp, "The Application of Segmentation and Grid Shock Absorber Concept for Reliable Power Grids," in $12^{\text {th }}$ International Middle-East System Conference, 2008.

[13] H. Clark, A. A. Edris, M. M. El-Gasseir and K. Epp, "Softening the blow of disturbances," IEEE Power \& Energy Magazine, pp. 30-41, Jan/Feb 2008.

[14] M. El-Gasseir and H. D. Kenneth, "Electricity market oriented DCSegmentation design and optimal scheduling for electrical power transmission", United States Patent 7620482, 17 November 2009.

[15] K. Sun , K. Hur and P. Zhang, "A New Unified Scheme for Controlled Power System Separation Using Synchronized Phasor Measurements," IEEE Transactions on Power Systems, vol. 26, no. 3, pp. 1544-1554, 2011.

[16] J. Wu, J. Wen, H. Sun and S. Cheng, "Feasibility Study of Segmenting Large Power System Interconnections With AC Link Using Energy Storage Technology," IEEE Transactions on Power Systems, vol. 27, no. 3, pp. 1245-1252, 2012.

[17] D. Woodford, "Segmentation - A Solution for the Future," High Powered Research for the Real World, Pulse, October 2008.

[18] L. Bizumic, R. Cherkaoui, O. Alizadeh Mousavi and A. Arestova, "HVDC Interconnections for Large Power Systems, the Path to the More Robust and Efficient Transmission Network," in CIGRE Symposium - The Electric Power System of the Future, Bologna, 2011.

[19] H. Huang, Z. Xu and X. Lin, "Improving Performance of MultiInfeed HVDC Systems Using Grid Dynamic Segmentation 
Technique Based on Fault Current Limiters," IEEE Transactions on Power Systems, vol. 27, no. 3, pp. 1664-1672, 2012.

[20] X. Fang and J. H. Chow, "BTB DC link modeling, control, and application in the segmentation of AC interconnections," in IEEE Power \& Energy Society General Meeting, Calgary, 2009.

[21] R. Leelaruji, L. Vanfretti, M. Ghandhari and L. So der, "Coordination of protection and VSC-HVDC systems for mitigating cascading failures," in International Conference on Power System Technology, Hangzhou, 2010.

[22] J. Pan, R. Nuqui, L. Tang and P. Holmberg, "VSC-HVDC Control and Application in Meshed AC Networks," in IEEE-PES General meeting, Pittsburgh, 2008.

[23] B. A. Carreras, D. E. Newman, I. Dobson and A. B. Poole , "Evidence for self-organized criticality in a time series of electric power system blackouts," IEEE Transactions Circuits and Systems, vol. 51, no. 9, pp. 1733-1740, 2004.

[24] "European Network of Transmission System Operators for Electricity, Monthly Statistics 2002-2012," ENTSOE, July 2012. [Online]. https://www.entsoe.eu/resources/publications/generalreports/monthly-statistics/.

[25] A. Clauset, C. R. Shalizi and M. E. Newman, "Power-law distributions in empirical data," SIAM Review, vol. 51, no. 4, pp. 661-703, 2009.

[26] M. Rosas-Casals and R. Solé, "Analysis of major failures in Europe's power grid," Electrical Power and Energy Systems, vol. 33, pp. 805-808, 2011.

[27] D. E. Newman, B. A. Carreras, V. E. Lynch and I. Dobson, "Exploring complex systems aspects of blackout risk and mitigation," IEEE Transactions on Reliability, vol. 60, no. 1, pp. 134-143, 2011

[28] "Initial review of methods for cascading failure analysis in electric power transmission systems," in IEEE Power Engineering Society General Meeting, Pittsburgh, 2008.

[29] O. Alizadeh Mousavi, R. Cherkaoui and M. Bozorg, "Blackouts Risk Evaluation by Monte Carlo Simulation Regarding Cascading Outages and System Frequency Deviation," Electric Power Systems Research, vol. 89, no. 1, pp. 157-164, 2012.

[30] H. Ren, I. Dobson and B. A. Carreras, "Long-Term Effect of the n-1 Criterion on Cascading Line Outages in an Evolving Power Transmission Grid," IEEE Transactions on Power Systems, vol. 23, no. 3, pp. 1217-1225, 2008.

[31] R. Billinton, J. Otengadjei and R. Ghajar, "Comparison of 2 alternate methods to establish an interrupted energy assessment rate," IEEE Transactions on Power Systems, vol. 2, no. 3, pp. 751$757,1987$.

[32] J. M. Carlson and J. Doyle, "Highly Optimized Tolerance: A Mechanism for Power Laws in Designed Systems," Physical Review, vol. 60, no. 2, pp. 1412-1427, 1999. 